\title{
The Value of Serum Amyloid A on Early Diagnosing and Prognosis for Perioperative Patients with Extracorporeal Circulation
}

\author{
B. MA, L. HE ${ }^{1}$, Y. XIA², L. CHI, Z. PIAO, X. SUN, J. DAI, C. YANG AND F. SHEN ${ }^{1 *}$ \\ Department of ICU, ${ }^{1}$ Department of Cardiology, ${ }^{2}$ Department of Cardiac Surgery, Zhejiang Greentown Cardiovascular \\ Hospital, Hangzhou 310000, China
}

Ma et al.: Serum Amyloid A in perioperative patients with extracorporeal circulation

The present study was aimed to investigate the value of serum amyloid $A$ in the early diagnosis of infection in patients with extracorporeal circulation and to evaluate the prognosis of patients. This was a prospective observational study. Patients admitted to the intensive care unit after cardiopulmonary bypass surgery between January 2017 and January 2019 were continuously collected. Daily blood serum amyloid $A$ and traditional inflammatory markers (white blood cell, C-reactive protein and procalcitonin) were continuously monitored 1 day before surgery and 3 days after surgery. The efficacy of serum amyloid $A$ and traditional inflammatory indicators in the early diagnosis of infection was assessed by receiving operating characteristic curve, and the efficacy of serum amyloid $A$ in predicting the prognosis of patients was assessed by multivariate logistic regression analysis. A total of 150 patients admitted to ICU after extracorporeal circulation was included in the study period. There were 58 patients with perioperative infection and 92 patients in the non-infected group. There were 18 patients in the death group, accounting for $12 \%$. Serum amyloid $A$ and traditional inflammatory indicators were increased in all patients on the first day after surgery compared with that before surgery, but in the non-infected group and the survival group, the decrease rate was significantly faster than that in the infected group and the death group within three days after surgery, $p<0.05$. According to ROC curve analysis, the diagnostic efficacy of perioperative infection was serum amyloid A, C-reactive protein, procalcitonin and white blood cell respectively, and the sensitivity and specificity were 84.48 and $86.96,79.31$ and $89.13,65.52$ and $98.91,89.66$, and $71.74 \%$, respectively. Multivariate logistic regression analysis indicated that the blood serum amyloid $A$ level on the first day after surgery was an independent risk factor for hospitalization and death, OR was 1.49 $(95 \%$ CI was 1.27-2.96, $p<0.01)$. Serum amyloid A could be used for early diagnosis of perioperative infection after extracorporeal circulation, and is an independent risk factor for prognosis of patients, and its value is better than traditional inflammatory indicators.

Key words: SAA, extracorporeal circulation, infection 
With the development of society, the aging of population has come. Cardiovascular diseases have become the main diseases affecting human health and life $\operatorname{span}^{[1,2]}$. More and more patients need coronary artery bypass grafting, valve replacement and aortic dissection surgery, which need the support of cardiopulmonary bypass. During cardiopulmonary bypass, components of the blood system are destroyed and inflammatory mediators are activated. Subsequent reperfusion injury, such as reperfusion, rewarming and so on, will cause different degrees of inflammatory activation in patients after cardiopulmonary bypass surgery and the inflammatory indicators will increase ${ }^{[3-5]}$. For clinicians, it is a very common clinical problem to judge whether there is infection after operation and whether antibiotics are needed. Traditional inflammatory indicators such as white blood cell (WBC), C-reactive protein (CRP) and procalcitonin (PCT) alone cannot meet the clinical guidance of antiinfective therapy for patients after cardiopulmonary bypass. Some studies have found that serum amyloid A (SAA) can more accurately judge infection, but it has not been used in patients after cardiopulmonary bypass surgery ${ }^{[6-8]}$. Therefore, this study was to explore whether SAA can effectively diagnose infection after cardiopulmonary bypass and predict the prognosis of patients.

This study was a prospective observational study. The research period was from January 2017 to January 2019 in the Zhejiang Greentown Cardiovascular Hospital. Cardiopulmonary bypass patients who met the inclusion criteria were collected. Inclusion criteria were, patients $18 \mathrm{y}$ or older than $18 \mathrm{y}$; patients underwent cardiopulmonary bypass; patients were admitted to ICU after operation. Exclusion criteria were, patients or family members who refuse to participate in the study were excluded; patients who have cancer or were at endstage were excluded; patients whose hospitalization time was less than $7 \mathrm{~d}$ were excluded, patients who have rheumatoid immune diseases and kidney diseases were excluded. The informed consent signed by the patients or their family members was obtained and this study was approved by the medical ethics committee of Zhejiang Greentown Cardiovascular Hospital.

Cardiopulmonary bypass patients who met the inclusion criteria were collected in this study. Clinical information collected were patient's gender, age, preoperative diagnosis, cardiac function grading, SAA, WBC, CRP,
PCT, bedside chest X-ray, B-mode ultrasound, blood culture, urine routine and so on, $1 \mathrm{~d}$ before operation and 1,2 and $3 \mathrm{~d}$ after operation. Patients with perioperative infection or not were judged by a group of physicians with more than two deputy chief physicians in the ward after comprehensive disease information and antiinfection program was formulated. If there were difficulties in diagnosis, the specialists in nosocomial infections department should be consulted in time. CRP was detected using Hitachi 27060 (Brahms Company of Germany), the PCT detector and kit from Brahms Company of Germany (Brahms PCT-Q), and SAA was detected by immune transmission turbidimetry (Hitachi 7170A Automatic Biochemical Analyzer).

Normality test was carried out on measurement data. Normality test of measurement data shows that normal distribution was expressed by mean \pm standard deviation, non-normal distribution was expressed by median and inter quartile range, and counting data was expressed by ratio or rate. The measurement data of two groups of normal distribution were compared by $t$ test, the measurement data of non-normal distribution were compared by Mann-Whitney U test, and the ratio was compared by $\mathrm{x}^{2}$ test. The variation trend of inflammatory markers in different groups was evaluated by repeated measurement variance analysis. The ROC curve was used to calculate the efficacy of inflammation indicators in judging perioperative infection. Multivariate logistic regression analysis was used to analyze the independent risk factors affecting hospitalization mortality. SPSS 22.0 software (SPSS Inc., Chicago, IL) was used for all statistical processing and mapping, with $\mathrm{p}<0.05$ as the significant difference.

A total of 150 patients, aged 58-89 y, were enrolled in the study period, with an average age of $71.5 \pm 12.4 \mathrm{y}$. There were 81 males $(54 \%)$ and 69 females (46\%). There were 58 cases $(38.7 \%)$ of perioperative infection, 39 cases $(67.2 \%)$ of which were pulmonary infection, 10 cases $(17.2 \%)$ of which were bloodstream infection, 4 cases $(6.9 \%)$ of surgical wound infection, 3 cases $(5.2 \%)$ of urinary tract infection and 2 cases $(3.5 \%)$ of intestinal infection. There was no significant difference in gender, age, preoperative diagnosis and cardiac function grade between the infected group and the noninfected group ( $>0.05$ ). Eighteen patients died during hospitalization, with a mortality rate of $12 \%$. The main causes of death were multiple organ failure and sepsis. 

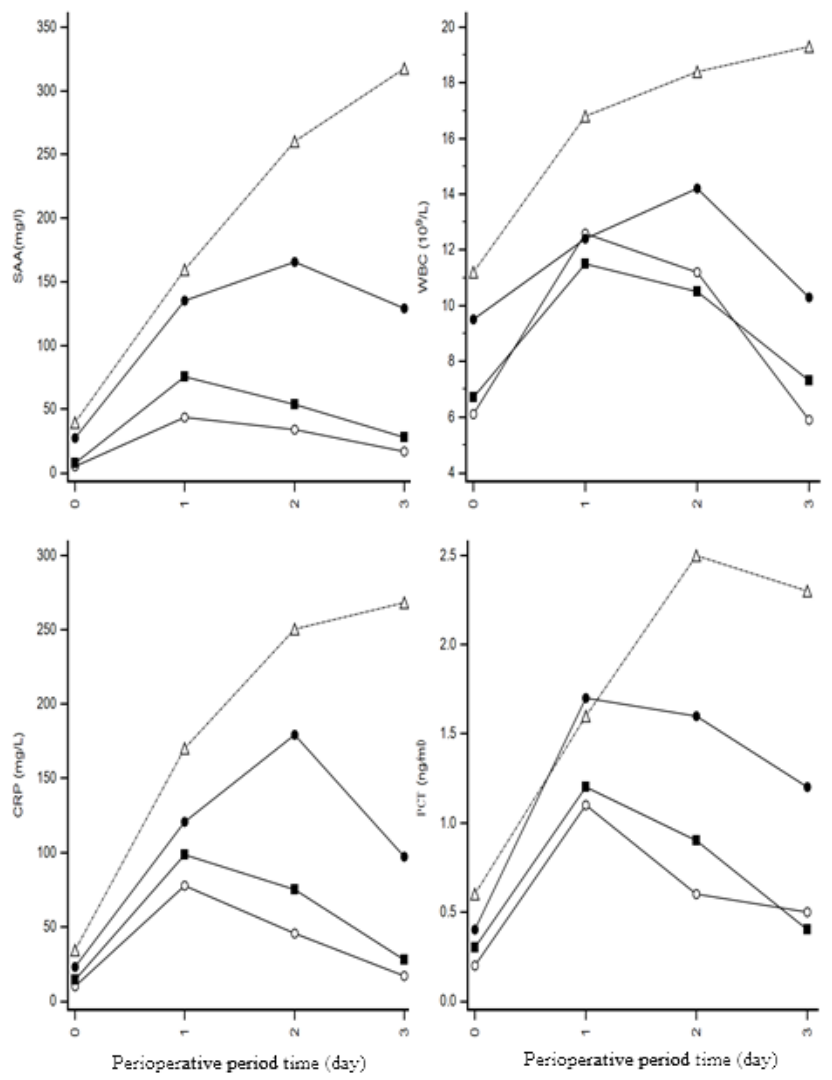

Fig. 1: Changes of SAA and traditional inflammatory markers during perioperative period in patients undergoing cardiopulmonary bypass

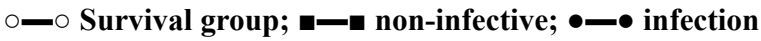
group; $\Delta-\Delta$ death group

Fig. 1 describes the changes of SAA and traditional inflammatory markers (WBC, CRP and PCT) during perioperative period in infected and non-infected patients, dead and surviving patients. It can be seen that for all patients undergoing cardiopulmonary bypass, the inflammation index on the first day after operation was higher than that before operation. After repeated measurements of variance analysis, SAA and WBC, CRP and PCT in non-infected group decreases significantly faster than those in infected group, and $\mathrm{F}$ values were 133.6, 90.2, 117.5 and 76.8, respectively, $p<0.05$. SAA and traditional inflammatory markers (WBC, CRP and PCT) does not show a significant downward trend (upward trend) within $3 \mathrm{~d}$ after operation in the dead group. There were significant differences in the trend of index change between death group and survival group, and F values were 172.4, 98.6, 144.0 and 82.1, respectively, $\mathrm{p}<0.05$.

The results of ROC curve analysis showed that SAA, WBC, CRP and PCT in blood on the second day after operation could differentiate infection from noninfection. The diagnostic efficacy was SAA, CRP, PCT and $\mathrm{WBC}$ in turn. The sensitivity was $84.48,79.31$,
$65.52,89.66 \%$, respectively. The specificity was 86.96 , $89.13,98.91$, and $71.74 \%$, respectively. The critical values were $118 \mathrm{mg} / 1,81 \mathrm{mg} / 1,1.4 \mathrm{ng} / \mathrm{ml}$ and $10.7 \times 10^{9} / 1$, respectively. Fig. 2 describes and compares ROC curves of different indicators for diagnosis of infection. Statistical comparison shows that SAA was superior to $\mathrm{WBC}$ and PCT in the diagnosis of infection ( $\mathrm{P}$ was 0.03 and 0.04, respectively), which were equivalent to CRP, $\mathrm{p}=0.13$. There was no significant difference between the other 2 groups ( $p>0.05$; Table 1 and fig. 2 ).

Gender, age, preoperative diagnosis and preoperative cardiac function were graded. APACHEII score was performed within $24 \mathrm{~h}$ of admission to ICU. APACHEII score within $24 \mathrm{~h}$ after admission to ICU, infection, SAA and traditional inflammatory markers on the first day after operation were recorded. Serum SAA and traditional inflammation indices on the first day after operation were taken as covariates. Multivariate logistic regression analysis was performed for infection and mortality. The results showed that the level of SAA in blood on the first day after operation was an independent risk factor for hospitalization mortality. OR was 1.49 (95\% CI is 1.27-2.96, $\mathrm{p}<0.01$ ). In addition, patients' age, APACHEII score and cardiac function grading were also risk factors for hospital mortality (Table 2).

SAA is an acute phase reactive protein, highly conserved in vertebrates and belongs to the heterogeneous proteins of apolipoprotein family with a fraction of 13581 . The human SAA gene is located on chromosome 11, and the liver is the main site for SAA synthesis. In addition,

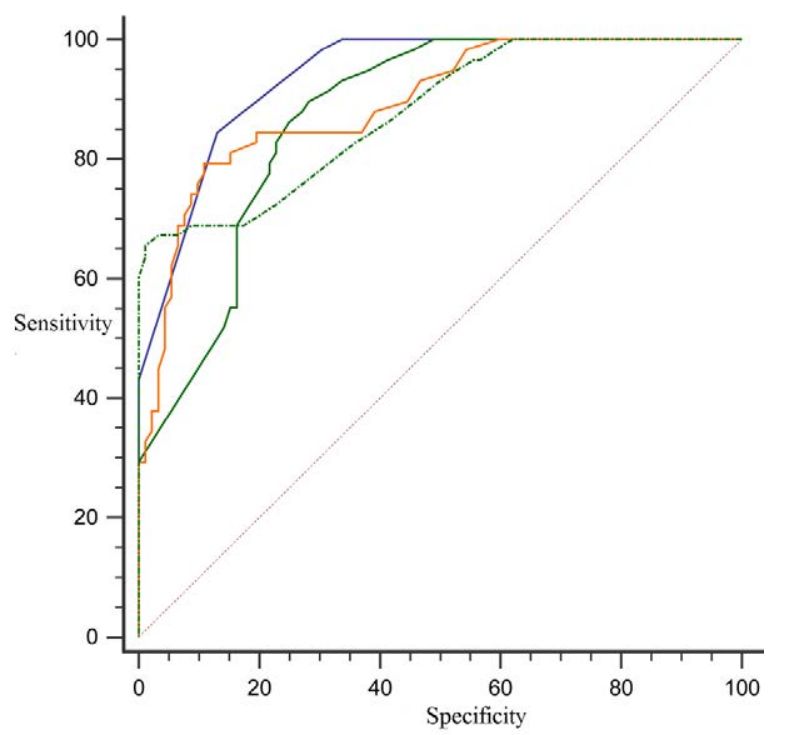

Fig. 2: ROC curves of SAA and traditional inflammatory markers in patients after cardiopulmonary bypass

SAA and traditional inflammatory markers for evaluating infection in patients after cardiopulmonary bypass $-\mathrm{SAA}$; WBC; - CRP; --- PCT 
TABLE 1: SAAAND TRADITIONAL INFLAMMATORY MARKERS IN INFECTION AFTER CARDIOPULMONARY BYPASS

\begin{tabular}{lcccccccc}
\hline Index & $\begin{array}{c}\text { Critical } \\
\text { value }\end{array}$ & AUC & Sensitivity & Specificity & $\begin{array}{c}\text { Positive } \\
\text { likelihood ratio }\end{array}$ & $\begin{array}{c}\text { Negative } \\
\text { likelihood ratio }\end{array}$ & $\begin{array}{c}\text { Positive } \\
\text { predictive value predictive value }\end{array}$ \\
\hline SAA & $118 \mathrm{mg} / \mathrm{l}$ & 0.937 & 84.48 & 86.96 & 6.48 & 0.18 & 80.3 & 89.9 \\
WBC & $10.7 \times 10^{9} / \mathrm{l}$ & 0.872 & 89.66 & 71.74 & 3.17 & 0.14 & 66.7 & 91.7 \\
CRP & $81 \mathrm{mg} / \mathrm{l}$ & 0.893 & 79.31 & 89.13 & 7.3 & 0.23 & 82.1 & 87.1 \\
PCT & $1.4 \mathrm{ng} / \mathrm{ml}$ & 0.877 & 65.52 & 98.91 & 60.28 & 0.35 & 82.4 \\
\hline
\end{tabular}

TABLE 2: MULTIVARIATE LOGISTIC REGRESSION ANALYSIS OF HOSPITALIZATION MORTALITY AFTER CARDIOPULMONARY BYPASS

\begin{tabular}{lccc}
\hline Risk factors & OR & $95 \% \mathrm{Cl}$ & $\mathrm{P}$ \\
\hline Age & 1.08 & $1.04-1.22$ & 0.02 \\
APACHEll score & 1.79 & $1.41-4.33$ & $<0.001$ \\
Classification of cardiac function & 1.62 & $1.08-2.54$ & $<0.001$ \\
SAA on the first day after & 1.49 & $1.27-2.96$ & $<0.001$ \\
operation & & &
\end{tabular}

a small part of the human SAA gene is synthesized outside the liver ${ }^{[9-11]}$. Macrophages and monocytes are activated to release inflammatory mediators IL-1 and TNF- $\alpha$, which then stimulate stromal cells to express inflammatory mediators IL-6, IL-8 and monocyte chemokines when the body is subjected to acute stress such as infection, burns, trauma and surgery. These inflammatory mediators, especially IL- 1 and IL-6, enter the blood circulation and are transported to the liver, which stimulates the synthesis of SAA by hepatocytes and hepatic macrophages. At this time, the level of SAA in circulating blood increases significantly ${ }^{[12,13]}$.

SAA in circulating blood acts as a chemoattractant to induce monocytes, granulocytes, mast cells and $\mathrm{T}$ lymphocytes to reach the inflammation site. The antiinflammatory activities of SAA include inhibition of lymphocyte function, inhibition of TNF- $\alpha$ and IL-1mediated fever and inhibition of platelet aggregation. SAA can also inhibit the respiratory burst of neutrophils and prevent oxidative damage of tissues and cells ${ }^{[14,15]}$. Like CRP, SAA concentration is a sensitive indicator of early inflammation in infectious diseases, which is helpful for the diagnosis, monitoring and treatment of inflammation. PCT consists of 166 amino acids and is a precursor of calcitonin, which is mainly produced by thyroid medullary $\mathrm{C}$ cells. In healthy people, PCT gene (calcitonin gene-related peptide 1, CALC-1) is transcribed and translated.

PCT is produced only in thyroid medullary C cells. The PCT produced is quickly cleaved into three substances by special enzymes: calcitonin, calcitonin and N-terminal amino acid fragments. Only a small amount of PCT is released into the blood circulation system, and its concentration is generally less than
$0.05 \mathrm{ng} / \mathrm{ml}$. Some studies have shown that when inflammation occurs in the body, target cells (peripheral blood mononuclear cells) secrete PCT under the action of many inflammatory related factors such as lipopolysaccharide. PCT synthesis is greater than decomposition. Undecomposed PCT is released into the blood, which ultimately leads to the increase of PCT in the blood circulation. Current studies have supported that PCT is an infection-related marker and an ideal marker for the diagnosis of infection, with high sensitivity and specificity ${ }^{[16,17]}$.

It was found that SAA and traditional inflammatory markers increase in different degrees after cardiopulmonary bypass, mainly due to the destruction of blood system components and the activation of inflammatory mediators during cardiopulmonary bypass, as well as reperfusion injury mediated by reperfusion and rewarming after cardiopulmonary bypass. However, through ROC curve analysis, SAA was superior to other inflammatory indicators in the diagnosis of perioperative infection in patients undergoing cardiopulmonary bypass. The possible reason is that SAA is more sensitive to infection stimuli than acute phase proteins such as CRP.

At present, there are many studies on SAA at home and abroad, mainly focusing on early diagnosis of infection and early warning of prognosis of related diseases ${ }^{[18-20]}$. For example, SAA can be used as a prognostic indicator of coronary artery disease and cardiovascular disease in women. There is an association between SAA and epidemic coronary heart disease. The diagnostic value of SAA in unstable angina pectoris, the changes of SAA level and its clinical significance in patients with rheumatoid arthritis, the effects of continuous blood purification on SAA expression and $\mathrm{T}$ lymphocyte subsets in patients with multiple organ dysfunction syndrome, and the study on acute exacerbation of chronic obstructive pulmonary disease and kidneyrelated diseases were studied.

It was found that SAA on the first day after surgery could better assess the prognosis of patients, and was an independent risk factor for adverse prognosis of 
patients. It was also found that age, APACHEII score and cardiac function grading were independent risk factors for poor prognosis. In this group, the SAA level continues to rise in the dead group after operation, which might be related to the serious underlying diseases, poor immune function and serious infection after operation. There were some deficiencies in this study. Firstly, this study was a single-center observational study, and the extrapolation of the results was inevitably limited. Secondly, this study only monitors the changes of inflammatory markers within $3 \mathrm{~d}$ of perioperative period, and could not predict the changes of follow-up indicators. Finally, this study does not further explore the relationship between inflammatory markers and the severity of infection. To sum up, the prospective observational study found that SAA could be an early diagnose perioperative infection after cardiopulmonary bypass. Dynamic monitoring of SAA and traditional inflammation indicators could better diagnose patients with or without infection. The diagnostic value of SAA was better than traditional inflammation indicators. Postoperative SAA level was an independent risk factor for prognosis.

\section{REFERENCES}

1. Cong M, Zhao W, Liu T. Protective effect of human serum amyloid $\mathrm{P}$ on $\mathrm{CCl} 4$-induced acute liver injury in mice. Int $\mathrm{J}$ Mol Med 2017;40(2):454.

2. Płóciniczak A, Dzięgielewska-Gęsiak S, Brożek A, Blacha A, Nowicki M, Formanowicz D. High sensitivity C-reactive protein as a cardiovascular risk marker in independent community-living elderly persons. J Biol Regul Homeost Agents 2018;32(5):1199-204.

3. Türer ÇC, Ballı U, Güven B, Fetuin-A. serum amyloid A and tumor necrosis factor alpha levels in periodontal health and disease. Oral Dis 2017;23(3):379.

4. Lee PT, Bird S, Zou J. Phylogeny and expression analysis of C-reactive protein (CRP) and serum amyloid-P(SAP) like genes reveal two distinct groups in fish. Fish Shellfish Immunol 2017;65:42-51.

5. Salis S, Mazzanti VV, Merli G. Cardiopulmonary bypass duration is an independent predictor of morbidity and mortality after cardiac surgery. J Cardiothoracasc Vasc Anesth 2008;22(6):814-22.

6. Yuan H, Huang J, Lv B. Diagnosis value of the serum amyloid A test in neonatal sepsis: a meta-analysis. Biomed Res Int 2013;2013:520294.

7. Hedegaard SS, Wisborg K, Hvas AM. Diagnostic utility of biomarkers for neonatal sepsis--a systematic review. Infect Dis 2015;47(3):117-24.

8. Arnon S, Litmanovitz I, Regev RH. Serum amyloid A: an early and accurate marker of neonatal early-onset sepsis. J Perinatol 2007;27(5):297-302.

9. Yamada T. Serum amyloid A(SAA)-pathogenicity and implication of appearance in plasma. Rinsho Byori 2006;54(5):509-12.

10. Kusnierz-Cabala B, Galicka-Latala D, Naskalski JW. Diagnostic value of serum amyloid A protein (SAA) determination. Przegl Lek 2007;64(2):115-7.

11. Perek B, Kowalska K, Kempisty B, Nowicki A, Jankowski M, Nawrocki MJ, et al. Role of macrophages in the pathogenesis of atherosclerosis and aortocoronary graft disease. J Biol Regul Homeost Agents 2018;32(5):1055-9.

12. Wu TL, Tsai IC, Chang PY. Establishment of an in-house ELISA and the reference range for serum amyloid A (SAA): complementarity between SAA and C-reactive protein as markers of inflammation. Clin Chim Acta 2007;376(1-2):72-6.

13. Abe-Dohmae S, Kato KH, Kumon Y. Serum amyloid A generates high density lipoprotein with cellular lipid in an ABCA 1- or ABCA7-dependent manner. J Lipid Res 2006;47(7);1542-50.

14. Bjorkman L, Karlsson J, Karlsson A. Serum amyloid A mediates human neutrophil production of reactive oxygen species through a receptor independent of formyl peptide receptor like-1. J Leukoc Bioh 2008;83(2):245-53.

15. Hatanaka E, Furlaneto CJ, Ribeiro FP. Serum amyloid A-induced mRNA expression and release of tumor necrosis factor-alpha (TNF-alpha) in human neutrophils. Immunol Lett 2004;91(1):33-7.

16. Kasperska-Zajac A, Grzanka A, Machura E. Analysis of procalcitonin and CRP concentrations in serum of patients with chronic spontaneous urticaria. Inflamm Res 2013;62(3):30912.

17. Oberhofer D, Juras J, Pavicic AM. Comparison of C-reactive protein and procalcitonin as predictors of postoperative infectious complications after elective colorectal surgery. Croat Med J 2012;53(6):612-9.

18. Liuzzo G, Biasucci LM, Gallimore JR. The prognostic value of C-reactive protein and serum amyloid a protein in severe unstable angina. N Engl J Med 1994;18;331(7):417-24.

19. Jousilahti P, Salomaa V, Rasi V. The association of c-reactive protein, serum amyloid a and fibrinogen with prevalent coronary heart disease--baseline findings of the PAIS project. Atherosclerosis 2001;156(2):451-6.

20. Gür A, Oguzturk H, Köse A. Prognostic Value of Procalcitonin, CRP, Serum Amyloid A, Lactate and IL-6 Markers in Liver Transplant Patients Admitted to ED with Suspected Infection. In Vivo 2017;31(6):1179-85.

This is an open access article distributed under the terms of the Creative Commons Attribution-NonCommercial-ShareAlike 3.0 License, which allows others to remix, tweak, and build upon the work non-commercially, as long as the author is credited and the new creations are licensed under the identical terms

This article was originally published in a special issue: Special issue on "Drug Development and Human Health in China"

Indian J Pharm Sci 2020:82(1)spl issue2;26-30 